\title{
131I SPECT/CT in the Follow-up of Differentiated Thyroid Carcinoma: Incremental Value Versus Planar Imaging
}

Angela Spanu, Maria E. Solinas, Francesca Chessa, Daniela Sanna, Susanna Nuvoli, and Giuseppe Madeddu Department of Nuclear Medicine, University of Sassari, Sassari, Italy

Planar ${ }^{131}$ I scintigraphy is routinely used to detect radioiodineavid metastases of differentiated thyroid carcinoma (DTC). However, the modality has limitations, such as low sensitivity and lack of anatomic landmarks. We investigated whether SPECT with integrated low-dose CT may have additional value over planar imaging in detecting residue and metastases in DTC patients. Methods: We studied 117 consecutive thyroidectomized DTC patients. On 2 different hybrid dual-head $\gamma$-cameras (55 patients on one camera and 62 on the other), 108 patients underwent ${ }^{131}$ I diagnostic imaging and SPECT/ $\mathrm{CT}$, and 9 underwent posttherapeutic ${ }^{131}$ I planar imaging and SPECT/CT. We assigned an incremental value to SPECT/CT when it provided better identification and interpretation of the foci of radioiodine uptake, more correct anatomic localization and characterization, and precise differentiation between tumor lesions and physiologic uptake. Results: Planar imaging showed 116 foci of uptake in 52 of 117 patients, and SPECT/CT showed 158 foci in 59 of 117 patients, confirming all foci seen on planar imaging but identifying an additional 28 occult foci in 10 of 52 patients. Fourteen occult foci were shown on SPECT/CT in 7 further patients whose planar imaging findings were negative. SPECT/ CT correctly characterized 48 foci unclear on planar imaging, also defining location and extent. SPECT/CT was a determinant in classifying as neoplastic those foci for which planar imaging seemed to exclude malignancy, discriminating between residue and lymph node metastases in the neck, some of which were adjacent to salivary glands and had been missed on planar imaging. SPECT/CT also showed occult lesions in the mediastinum, abdomen, and pelvis and identified small bone metastases unsuspected on planar imaging. Globally, SPECT/CT had an incremental value over planar imaging in $67.8 \%$ of patients, modified therapeutic management in $35.6 \%$ of positive cases, and avoided unnecessary treatment in $20.3 \%$ of patients with only single benign lesions or physiologic uptake. Conclusion: ${ }^{131}$ I SPECT/CT improved planar data interpretation, showing a higher number of DTC lesions, more precisely localizing and characterizing DTC foci, and more correctly differentiating between physiologic uptake and metastases, thus permitting the most appropriate therapeutic approach to be chosen. A wider use of this method is suggested complementary to planar imaging in selected DTC patients.

Received Aug. 1, 2008; revision accepted Oct. 23, 2008.

For correspondence or reprints contact: Giuseppe Madeddu, Department of Nuclear Medicine, University of Sassari, Viale San Pietro, 807100 Sassari, Italy.

E-mail: giuseppe.madeddu@email.it

COPYRIGHT ๑ 2009 by the Society of Nuclear Medicine, Inc.
Key Words: oncology; SPECT/CT; ${ }^{131} \mathrm{I}$; differentiated thyroid cancer

J Nucl Med 2009; 50:184-190

DOI: 10.2967/jnumed.108.056572

$C$ onventional planar ${ }^{131}$ I whole-body scintigraphy, in association with serum thyroglobulin measurement, is still considered the routine diagnostic procedure in patients with well-differentiated thyroid carcinoma (DTC). This modality is used in the detection of both thyroid tissue residue and local and distant metastases, after thyroidectomy for staging and after radioiodine ablation for restaging and long-term follow-up (1). A sensitivity of $45 \%-75 \%$ and a specificity of $90 \%-100 \%$ have been reported in the literature for diagnostic planar ${ }^{131} \mathrm{I}$ whole-body imaging in detecting recurrences or metastases from DTC (2-9).

Some factors can limit the performance of this procedure. The limited resolution of the general-purpose $\gamma$-camera when used for planar acquisitions, together with background activity in iodine images, can give false-negative results for small lesions, whereas the presence of numerous areas of physiologic uptake not always easily differentiable from pathologic uptake can give false-positive results $(6,10-12)$. Planar imaging, not giving landmarks for the pathologic focal uptake or therefore their exact anatomic site, can sometimes fail to indicate the correct disease classification and therapeutic management. False-negative results can occur on ${ }^{131}$ I whole-body imaging in poorly differentiated carcinoma, which shows a reduced or absent uptake capability but high thyroglobulin secretion. In such DTC carcinomas, besides conventional imaging methods such as ultrasound, CT, and MRI, other radioisotopic procedures have been useful, such as scintigraphy with the cationic lipophilic radiotracers ${ }^{99 \mathrm{~m}} \mathrm{Tc}-$ tetrofosmin and ${ }^{99 m}$ Tc-sestamibi $(13-17)$ and ${ }^{18} \mathrm{~F}-\mathrm{FDG}$ PET $(9,17,18)$ and ${ }^{18}$ F-PET/CT $(19-22)$, with ${ }^{18} \mathrm{~F}-\mathrm{FDG}$ taken up mainly by highly aggressive carcinomas.

SPECT has been proposed as a way to overcome the limitations of planar ${ }^{131}$ I whole-body imaging in iodineavid metastases. Although SPECT has higher sensitivity 
and better contrast resolution than planar acquisitions and can obtain cross-sectional scintigraphic images, the anatomic evaluation of lesion sites remains difficult.

The performance of SPECT may be further improved by fusing SPECT and CT images (23), either by using external or internal markers to coregister the 2 examinations performed in 2 different sessions $(24,25)$ or, more recently, by using integrated SPECT/CT systems (26-30) that permit simultaneous anatomic mapping and functional imaging. Both methods have the potential to determine the exact anatomic site of uptake, correctly characterizing those unclear on planar imaging and thus limiting false-positive results, in particular when using integrated methods in which the patient maintains the same position on the table during the entire examination. These methods can give information on the extent of the disease in addition to that provided by SPECT and CT separately.

In this study, we further investigated whether SPECT with low-dose CT may have additional value over conventional planar ${ }^{131}$ I whole-body imaging in detecting and characterizing thyroid tissue residue and local and distant metastases in thyroidectomized DTC patients and whether its contribution may modify the therapeutic strategy.

\section{MATERIALS AND METHODS}

\section{Patients}

One hundred seventeen consecutive patients, 28 men and 89 women aged 21-81 y who previously underwent total thyroidectomy for DTC (109 papillary, 7 follicular, 1 Hürthle cell), were prospectively studied. Of these patients, 108 underwent diagnostic ${ }^{131}$ I whole-body imaging (48-72 $\mathrm{h}$ after an orally administered dose of $185 \mathrm{MBq}$ ). In 9 of the 108, the imaging was performed about $40 \mathrm{~d}$ after thyroidectomy and before radioiodine therapy, while the patients were hypothyroidal. In 99 of the 108, the imaging was performed during long-term follow-up because of residue or suspected metastases after radioiodine ablation ( 1 dose in 82 patients, 2 doses in 12 patients, and $>2$ doses in 5 patients). Twenty-one of these 99 patients were hypothyroidal after 4-6 wk of withdrawal from L-thyroxin, and 78 had received an increment of exogenous thyroid-stimulating hormone (TSH) after recombinant human TSH (rh-TSH) stimulation (0.9 mg intramuscularly for 2 subsequent days, with euthyroidism maintained). The remaining 9 of 117 patients underwent posttherapeutic ${ }^{131} \mathrm{I}$ whole-body imaging 5-7 d after oral radioiodine administration $(3,700 \mathrm{MBq})$, with 6 being hypothyroidal after L-thyroxin discontinuance and 3 having undergone rhTSH stimulation.

Before scintigraphy, all patients underwent laboratory tests, such as the measurement of urinary iodine excretion (ioduria) and the assay of TSH, thyroglobulin, and antithyroglobulin antibodies, while hypothyroidal or after rh-TSH stimulation.

At scintigraphy, the TSH levels were always more than $50 \mu \mathrm{U} /$ $\mathrm{mL}$ and those of ioduria less than $300 \mu \mathrm{g} / \mathrm{L}$. The cutoff level of thyroglobulin was $0.2 \mathrm{ng} / \mathrm{mL}$.

Exclusion criteria included patients with non-radioiodine-avid neoplastic lesions but with high thyroglobulin levels as shown on ${ }^{99}$ Tc-tetrofosmin SPECT or ${ }^{18} \mathrm{~F}-\mathrm{FDG}$ PET and conventional imaging.
The protocol was in accordance with the Helsinki Doctrine on Human Experimentation, and the patients gave written informed consent before scintigraphy.

\section{Scintigraphy Protocol}

Planar ${ }^{131}$ I whole-body imaging was performed in both anterior and posterior projections using 2 variable-angle dual-head $\gamma$-cameras, the Millennium VG Hawkeye (GE Healthcare) in 55 patients and the more recent Infinia Hawkeye 4 (GE Healthcare) in 62 patients. High-energy, parallel-hole collimators were used, at a table speed of $10 \mathrm{~cm} / \mathrm{min}$ for a total time of $30 \mathrm{~min}(1,024 \times 256$ matrix). The imaging was completed with a spot view of selected body areas in anterior, posterior, and lateral projections (600 s/ view).

These $\gamma$-cameras are also part of hybrid systems equipped with an integrated $\mathrm{x}$-ray transmission system (low-dose CT) to provide anatomic maps for attenuation correction and image fusion. In both devices, the CT apparatus has a fixed-anode oil-cooled x-ray tube installed on the slip-ring gantry of the $\gamma$-camera and operates at $140 \mathrm{keV}$ and up to $2.5 \mathrm{~mA}$.

On these 2 hybrid systems, SPECT/CT was performed during the same session as planar whole-body imaging over the neck and chest with the patient in the same position, including the patient's arms. SPECT/CT was also focused on other suspected areas of increased uptake as seen on planar ${ }^{131} \mathrm{I}$ whole-body images. To minimize patient movement during acquisitions, in particular of the neck, we used special vacuum cushions to stabilize the position.

First, emission SPECT images were acquired over $360^{\circ}\left(180^{\circ}\right.$ per head) at the $364-\mathrm{keV}$ photopeak and $\pm 10 \%$ energy window, with the patient supine. A $128 \times 128$ matrix was used, with a $3^{\circ}$ angular step, an acquisition time of $30 \mathrm{~s}$ per frame $(30 \mathrm{~min}$ in total), and a zoom factor ranging from 1 to 1.2 according to the individual patient. The body contouring system was used to minimize the distance between the patient and the collimator.

The SPECT examination was followed by CT. In both devices, the $\mathrm{x}$-ray tube and detector array rotate together in a fixed geometry, at $2.6 \mathrm{rpm}$ for an $\mathrm{H}$-mode scan with the Millennium VG Hawkeye and at $2.0 \mathrm{rpm}$ for a $90^{\circ}$ L-mode scan with the Infinia Hawkeye 4. Multiple CT slices were obtained, in the transaxial mode when using the Millennium VG Hawkeye (a 10mm-thick slice in $13.8 \mathrm{~s}$, requiring 0.6 rotations and reconstructed online to a $256 \times 256$ image matrix) and in the helical mode when using the Infinia Hawkeye 4 (four 5-mm-thick slices obtained simultaneously with a beam coverage of $2 \mathrm{~cm}$ in each gantry rotation and reconstructed online to a $512 \times 512$ image matrix). CT scans were acquired within 10-12 min with the former device and within $4.5 \mathrm{~min}$ with the latter.

Cross-sectional attenuation images $(128 \times 128$ image matrix $)$ in which each pixel represents the attenuation of the imaged tissue were generated in all cases.

SPECT images were reconstructed with the iterative method and fused with CT images using a dedicated software package (Xeleris workstation; GE Healthcare).

\section{Data Analysis}

All images obtained by both planar acquisition and SPECT/CT were analyzed separately by 2 experienced nuclear medicine physicians who were unaware of the clinical findings, of any other diagnostic imaging data, and of the definitive histopathologic diagnosis. Tumor uptake of ${ }^{131}$ I was defined as any focal or 
diffuse uptake that was higher than the surrounding background and incompatible with physiologic activity. Foci of uptake in the salivary glands, urinary collecting system, and gastrointestinal tract were considered physiologic except for some small focal, circumscribed areas that could not be clearly distinguished and were considered suggestive of lesions on planar imaging.

Planar data were considered unclear when it was not easy to ascertain the anatomic site or to characterize the foci of uptake. An incremental value with respect to planar ${ }^{131} \mathrm{I}$ whole-body imaging was assigned to the SPECT/CT fusion images when they provided better identification and interpretation of the uptake foci, more correct anatomic localization and characterization, and precise differentiation between tumor lesions and physiologic uptake when not obtained from the planar images.

Interobserver variability was extremely low; disagreement was observed in only 3 cases in the analysis of SPECT/CT images and was resolved by consensus.

The gold standard for confirming the absence or presence of malignancy suspected on planar imaging and SPECT/CT was surgery with definitive histopathologic findings, clinical examination with changes in thyroglobulin levels, or radiologic follow-up for at least 6 mo. In patients with positive planar and SPECT/CT findings who underwent radioiodine therapy, posttherapeutic scan findings were also considered.

The neck was also studied by ultrasound in all patients and by pinhole SPECT with the cationic lipophilic ${ }^{99 \mathrm{~m}} \mathrm{Tc}$-tetrofosmin in 7 patients with detectable thyroglobulin serum levels and discordant findings on ${ }^{131} \mathrm{I}$ scintigraphy and ultrasound. Diagnostic CT was performed on 7 patients to better clarify radioiodine foci in the thorax or abdominal cavity, and bone scanning was performed on 2 patients in whom bone metastases were suspected.

\section{RESULTS}

Planar ${ }^{131}$ I imaging showed 116 foci of uptake in 52 of 117 patients: in 36 patients, the foci (60 foci) were identified only in the neck; in 4 patients, both in the neck ( 7 foci) and outside the neck (17 foci); and in the remaining 12 patients, only outside the neck (32 foci). SPECT/CT showed 158 foci in 59 of 117 patients, confirming all 116 foci seen on planar imaging in 52 of the patients but also identifying in 10 of these patients 28 occult foci ( 8 in the neck and 20 outside the neck). In 7 of 117 patients, SPECT/ CT found 14 additional foci ( 6 in the neck and 8 outside the neck) that had been occult on planar imaging.

In the neck, planar imaging and SPECT/CT showed 67 and 81 foci, respectively.

Fifty of 67 foci detected on planar imaging in 40 patients were classified as residue in the thyroid bed (33 foci) or in the thyroglossal tract (17 foci), and 17 were considered unclear. SPECT/CT showed all 67 foci, confirming 45 of 50 of these as residue, but changed the classification of the remaining 5 of 50 foci in 5 patients (thyroglobulin levels, $1.9-4.3 \mathrm{ng} / \mathrm{mL}$ ) from residue to metastatic locoregional lymph nodes, 2 of which were ultrasound-negative but 99mTc-tetrofosmin pinhole SPECT-positive and all of which were diagnosed at surgery. SPECT/CT well characterized the 17 unclear foci in 14 of the 40 patients, correctly classifying them as 8 cases of residue in the thyroid bed in
6 patients; 2 cases of locoregional metastatic lymph nodes found at surgery, which were the only lesions in 2 patients (thyroglobulin levels, 4.2 and $4.5 \mathrm{ng} / \mathrm{mL}$, respectively; both cases were negative on ultrasound but 1 was positive on ${ }^{99 m}$ Tc-tetrofosmin pinhole SPECT); 2 bone metastases in 1 patient (Fig. 1); and 5 circumscribed areas of physiologic uptake, which were the only foci in 5 patients with undetectable or borderline thyroglobulin. SPECT/CT identified a further 14 lesions occult on planar imaging: 9 cases of locoregional metastatic lymph nodes and 5 cases of residue in 11 patients. Five of these 11 patients showed negative findings on planar imaging but 6 foci on SPECT/ CT: 2 cases of residue, negative on ultrasound, in 2 patients (thyroglobulin levels, 0.2 and $1.9 \mathrm{ng} / \mathrm{mL}$, respectively) and 4 cases of metastatic lymph nodes, 2 of which were closely adjacent to a salivary gland, in the other 3 patients (thyroglobulin levels, 5.9-13.5 ng/mL). Three of 4 lymph nodes
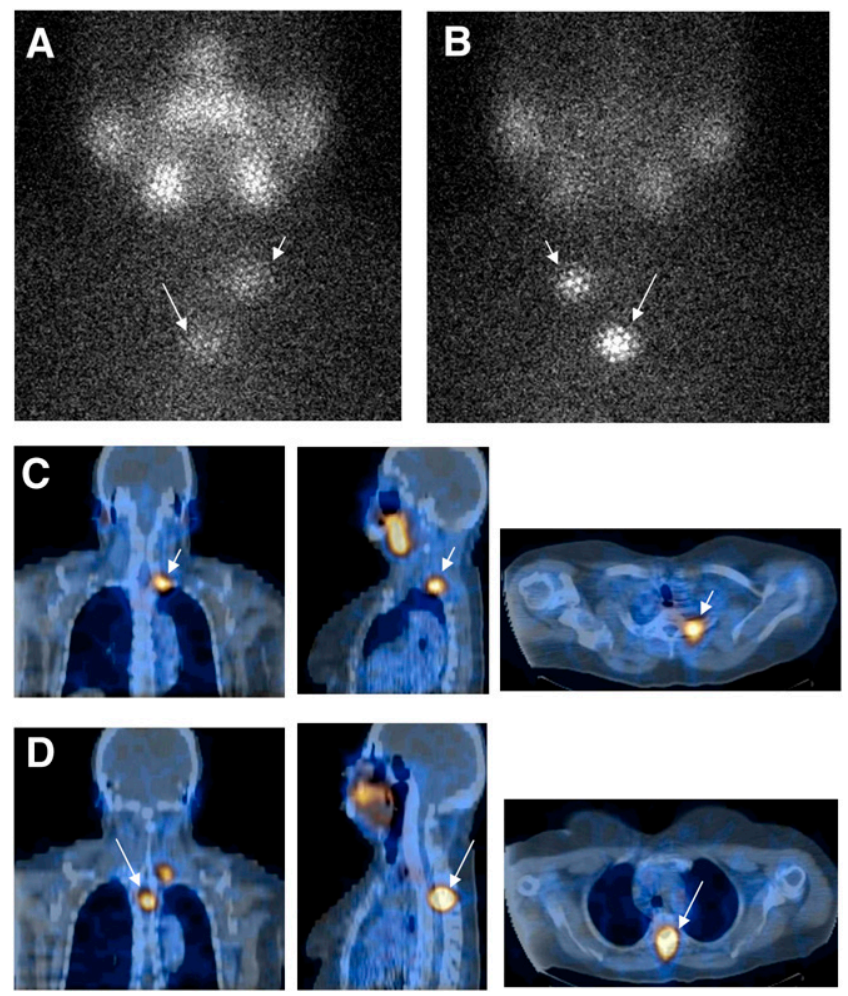

FIGURE 1. A 72-y-old female patient with thyroidectomized papillary thyroid carcinoma in follow-up. Planar ${ }^{131}$ I neck/upper thorax imaging in both anterior (A) and posterior (B) views showed 2 circumscribed foci of radioiodine uptake, 1 in the lower neck (short arrow), and the other in the upper thorax (long arrow), more evident in posterior view. Only SPECT/CT image fusion ( $C$ and $D$ ) in coronal, sagittal, and transverse slices identified anatomic site, suggesting that foci corresponded to radioiodine-avid bone metastases, with upper focus (short arrow) localized in first left costovertebral articulation (C) and lower focus (long arrow) in second thoracic vertebra (D). Thyroglobulin level after $\mathrm{rh}-\mathrm{TSH}$ was $3.5 \mathrm{ng} / \mathrm{mL}$. Diagnosis was confirmed on posttherapeutic radioiodine scintigraphy. 
were also positive on ultrasound, and the remaining lymph node was positive on ${ }^{99 \mathrm{~m}} \mathrm{Tc}$-tetrofosmin pinhole SPECT; all were confirmed at surgery. In the remaining 6 of 11 patients, SPECT/CT detected 8 further occult foci besides confirming 11 other foci classified as residue on planar imaging. Three of the 8 foci were residue, 1 of which was negative on ultrasound, and 5 of 8 were metastatic lymph nodes; all were confirmed at histology. Two of the lymph nodes (in 2 patients, with thyroglobulin levels of $0.4-5.6$ $\mathrm{ng} / \mathrm{mL}$, respectively) were negative or aspecific for malignancy on ultrasound but positive on ${ }^{99 \mathrm{~m}} \mathrm{Tc}$-tetrofosmin pinhole SPECT. Four lymph nodes were in the laterocervical region, and 1 was adjacent to a salivary gland (Fig. 2).

Outside the neck, planar imaging showed 49 foci in 16 patients and SPECT/CT showed 77 foci in 18 patients.
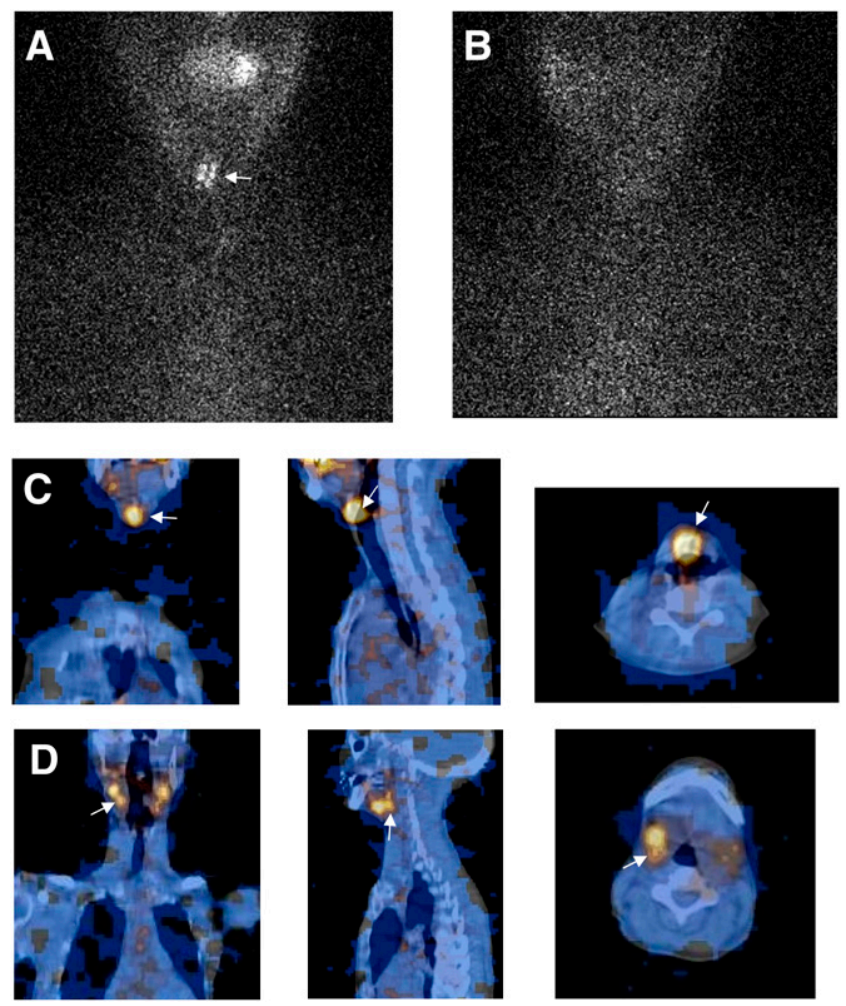

FIGURE 2. A 65-y-old woman with thyroidectomized papillary thyroid carcinoma in follow-up. Planar ${ }^{131}$ I imaging of neck and chest in both anterior (A) and posterior (B) views detected only 1 focus of radioiodine uptake, corresponding to residue in thyroglossal tract (arrow). SPECT/CT image fusion (C) in coronal, sagittal, and transverse slices confirmed residue (arrow) seen on planar imaging but also showed 1 radioiodine-avid lymph node metastasis (arrow, D) in right submandibular region closely adjacent to salivary gland. Ultrasound was considered aspecific for malignancy, and thyroglobulin serum level under hypothyroidal conditions after L-thyroxin withdrawal was $0.4 \mathrm{ng} / \mathrm{mL}$. Diagnosis was definitively confirmed at surgery, which was performed on the basis of SPECT/CT, revealing papillary thyroid carcinoma lymph node metastasis.
Both planar imaging and SPECT/CT detected 1 focus in the head/skull, but only SPECT/CT clarified that the focus was an area of physiologic uptake in the rhinopharynx.

In the thorax, SPECT/CT showed all 13 foci classified as pulmonary metastases on planar imaging in 7 patients, all with high thyroglobulin levels. However, although confirming the classification of 9 of 13 foci, SPECT/CT changed the diagnosis of the remaining 4 foci from pulmonary metastases to mediastinal lymph node metastases (in 2 foci) and from pulmonary metastases to thorax wall metastases with extension to bone (in the remaining 2) (Fig. 3). The latter 2 were also confirmed on diagnostic CT, and the radioiodine dose was more accurately defined. SPECT/CT also characterized 15 foci (in 10 patients) that had been unclear on planar imaging: Three of the 15 (in 1 patient) were metastatic lesions, 1 in the mediastinum and 2 in the ribs, with the latter 2 confirmed on bone scanning. Two of the 15 (in another patient, who was recovering from bronchitis) were benign bronchial mucous secretion (Fig. 4). Ten of the 15 (in the remaining 8 patients) were circumscribed areas of physiologic uptake, 6 of which represented the only foci. Seventeen further foci (in 5 patients) seen on SPECT/CT corresponded to malignant lesions occult on planar imaging (11 pulmonary metastases, 4 mediastinal lymph node metastases, and 2 rib metastases), and only 8 of these 17 were identified on diagnostic CT. Four of 5 patients (thyroglobulin levels, 184-3,500 ng/mL) also had other lesions positive on both planar imaging and SPECT/CT, whereas the remaining 1 patient (thyroglobulin level, $3.6 \mathrm{ng} /$ $\mathrm{mL}$ ), with 1 pulmonary and 2 mediastinal metastases, had negative findings on planar imaging.
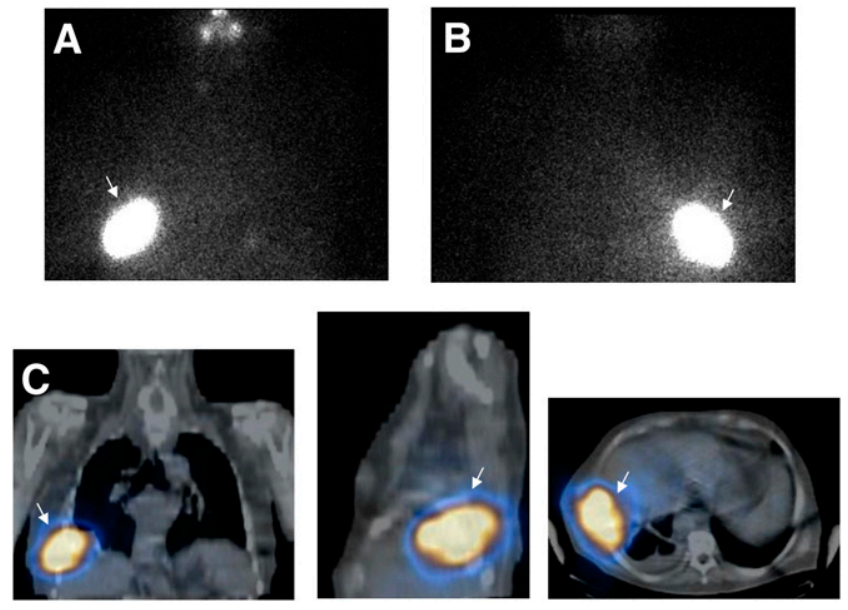

FIGURE 3. A 69-y-old man with thyroidectomized papillary thyroid carcinoma in follow-up. Planar ${ }^{131}$ I chest imaging in both anterior (A) and posterior (B) views showed wide, circumscribed focus of radioiodine uptake in lower lateral region of thorax on right (arrow). Only SPECT/CT image fusion (C) in coronal, sagittal, and transverse slices correctly established that focus corresponded to metastasis in thorax wall with extension to bone (arrow), as also shown on diagnostic CT. Thyroglobulin level was $3,551 \mathrm{ng} / \mathrm{mL}$. Diagnosis was confirmed on posttherapeutic ${ }^{131}$ I whole-body imaging. 
FIGURE 4. A 69-y-old woman with thyroidectomized papillary thyroid carcinoma in follow-up. Planar 131| chest imaging in both anterior (A) and posterior (B) views showed 2 foci of marked radioiodine uptake in right and left ileal regions (arrows), which were considered suspect for metastases although thyroglobulin level was less than $0.2 \mathrm{ng} / \mathrm{mL}$. Only SPECT/CT image fusion (C) in coronal, sagittal left and right, and transverse slices determined that these foci involved proximal bronchial tree bilaterally (arrows), as was also confirmed on diagnostic CT. At history taking, patient referred to recent acute bronchitis, from which patient was then recovering; thus, uptake was probably due to mucous excretion in inflammatory exudate in bronchial tree. During 12 mo of follow-up, thyroglobulin serum levels remained undetectable, with absence of clinical signs of disease, excluding metastases.
In the abdomen or pelvis, SPECT/CT showed 31 foci. Concordantly with planar imaging, SPECT/CT correctly classified 1 focus in 1 patient to be skin contamination, and SPECT/CT confirmed that 4 sites of uptake (in 1 patient) on planar imaging were soft-tissue pelvic metastases. However, SPECT/CT also showed that these 4 metastases extended to bone, as confirmed on diagnostic $\mathrm{CT}$, thus better defining the therapeutic radioiodine dose. SPECT/CT well characterized 15 foci that had been unclear on planar imaging, classifying 13 (in 3 patients) as malignant and the remaining 2 (in 2 other patients) as circumscribed areas of physiologic uptake, 1 of which was the only focus in the patient. SPECT/CT found 11 neoplastic lesions that had been occult on planar imaging in 3 patients, 2 of whom had a thyroglobulin level of less than $2,000 \mathrm{ng} / \mathrm{mL}$ and 1 of which had a thyroglobulin level of $3.5 \mathrm{ng} / \mathrm{mL}$. The latter patient, whose findings on planar imaging had been negative, had 5 small metastases in the lumbar spine. Only 2 of these had also been detected on bone scanning, and only 1 had been detected on CT.

Globally, planar imaging and SPECT/CT findings were concordantly negative in $49.6 \%$ of patients (Table 1), who were considered disease-free as confirmed by undetectable thyroglobulin levels and negative findings on conventional imaging. Findings were concordantly positive in $44.4 \%$ of patients, with SPECT/CT detecting more foci in $8.5 \%$ of patients. Findings were discordant in $6 \%$ of patients, in whom only SPECT/CT showed metastases.

SPECT/CT did not add further information with respect to planar imaging in 19 of the 59 patients with positive findings (32.2\%). SPECT/CT did, however, have incremental value, detecting occult lesions or defining the exact anatomic site, characterizing residue and malignant foci, or differentiating physiologic uptake from metastases unassessed by planar imaging in 40 of 59 patients $(67.8 \%)$. SPECT/CT led to modified therapeutic management in 21 of these 40 patients (52.5\%), corresponding to $35.6 \%$ (21/ 59 ) of the positive cases globally considered. In these 21 patients, SPECT/CT led to a more correct selection of surgery or radiometabolic therapy, even permitting for the latter a more appropriate therapeutic radioiodine dose in 6 patients.

Finally, SPECT/CT clarified the origin of benign lesions or areas of physiologic uptake in 20 of the foci that had been unclear on planar imaging in 16 of 59 patients, thus reducing the false-positive results. SPECT/CT even permitted the avoidance of unnecessary treatment in 12 of these 16 patients, 12 of whom (20.3\%) had only 1 focus.

\section{DISCUSSION}

Together with thyroglobulin serum level assay and conventional radiologic procedures, ${ }^{131}$ I scintigraphy is commonly accepted as a basic component of the diagnostic strategy in patients thyroidectomized for DTC. However, often neither planar nor SPECT acquisitions are able to correctly determine the site of the lesions for better characterization or to differentiate pathologic from physiologic uptake.

The recent application of new technology that combines functional and anatomic SPECT/CT images has improved the ability of planar ${ }^{131}$ I imaging to detect iodine-avid foci (24-31).

TABLE 1. Incremental Value of SPECT/CT with Respect to Planar Imaging in Relationship to Number of Foci of Radioiodine Uptake

\begin{tabular}{lc}
\hline $\begin{array}{c}\text { Planar and SPECT/CT } \\
\text { imaging }\end{array}$ & $\begin{array}{c}\text { No. of DTC patients } \\
(n=117)\end{array}$ \\
\hline Concordantly negative & $58(49.6 \%)$ \\
Concordantly positive & $52(44.4 \%)$ \\
With same number of foci on & $42(35.9 \%)$ \\
planar imaging and SPECT/CT & \\
With higher number of foci on & $10(8.5 \%)$ \\
SPECT/CT than on planar imaging & \\
Discordant & $7(6.0 \%)$ \\
Planar imaging negative and & \\
SPECT/CT positive & \\
\hline
\end{tabular}


Placement of external markers at specific positions on the patient's body to coregister SPECT and diagnostic multislice spiral CT scans acquired in 2 different sessions has been reported to improve the diagnosis of lesions in $88 \%$ of DTC patients, with respect to SPECT alone and CT alone (25). However, some artifacts can occur with this method because of an unavoidable, although minimal, difference in positioning between the SPECT session and the CT session. Another technical problem is that the external markers are manually defined.

By acquiring SPECT and CT images on a hybrid Millennium VG Hawkeye scanner in the same session and with the patient in the same position immediately after posttherapeutic planar ${ }^{131} \mathrm{I}$ whole-body imaging, some authors have partially solved positioning problems, reducing registration artifacts due to physiologic uptake and improving anatomic assignment. With this method, foci of uptake have been identified in $44 \%$ of patients, and important information for clinical interpretation has been obtained for $38 \%$ of all foci (27). Using the same type of hybrid system, other authors (28) have found fusion images to have additional value over planar imaging in $57 \%$ of DTC patients, changing the therapeutic strategy in $41 \%$ of patients who underwent a diagnostic study.

In the present study, we used, like these authors $(27,28)$, the Millennium VG Hawkeye, or we used the more recent Infinia Hawkeye 4, and we found more foci with these systems than with planar imaging. In $67.8 \%$ of patients, we obtained more precise anatomic localization in different regions, such as the neck, thorax, abdomen/pelvis, and skeleton; improved image interpretation; and more correctly differentiated malignant lesions from sites of physiologic uptake, such as the salivary glands, esophagus, gastrointestinal tract, and liver. In $35.6 \%$ of patients with positive findings, we were led to a more appropriate decision about therapeutic management. All lesions determined to be a presumptive tumor on SPECT/CT were confirmed to be malignant.

The information provided by SPECT/CT was relevant in 17 of our patients in whom the procedure identified occult lesions missed on planar imaging. In some cases, the disease could be reclassified, the most correct prognosis determined, and the most appropriate therapeutic strategy selected. SPECT/CT was particularly relevant in those patients in whom the occult foci in the neck were the only ones found. The procedure was also able to differentiate between residue in the thyroid bed and well-localized cervical lymph node metastases, permitting the choice of radioiodine therapy for the former and surgery for the latter. In addition, the extent of the surgery could be optimized and the diagnosis definitively confirmed. In studies of the neck, SPECT/CT characterized as neoplastic the foci for which planar findings, although positive, seemed to exclude malignancy. SPECT/ $\mathrm{CT}$ also revealed lymph node metastases adjacent to salivary glands. All of these metastases were missed on planar imaging because of interference from high physiologic uptake of radioiodine in the salivary glands.
The addition of SPECT/CT offered important advantages by also showing lymph node metastases in the mediastinum and in the abdomen or pelvis, especially when these were the only lesions, and by identifying unsuspected neoplastic lesions in bone (even small bones) that had not been shown or characterized on planar imaging. SPECT/CT also revealed bone invasion from thorax wall metastases and from softtissue metastases in the abdomen or pelvis, thus demonstrating an increasingly worse disease prognosis and indicating a need to increase the therapeutic dose of radioiodine over that which had been initially proposed after planar imaging alone.

The capability of SPECT/CT to define the anatomic sites of foci justifies the additional use of low-dose CT not only when planar ${ }^{131}$ I whole-body imaging misses foci but also when it detects foci but finds them to be nonneoplastic; SPECT/CT can show malignancy in these cases. In the present study, SPECT/CT was also a determinant in patients whose planar imaging findings were unclear. In these patients, fusion images could show that radioiodine uptake was due to neoplastic lesions in some cases but to nonneoplastic lesions (benign disease or physiologic uptake) in other cases, correctly characterizing 48 unclear foci. Thus, SPECT/CT reduced false-positive findings on planar imaging, improving specificity. These data became even more important when the benign lesion or physiologic uptake identified on SPECT/CT was single and represented the only area of uptake, since unnecessary treatment could be avoided. In the present study, because thyroglobulin levels were also low or borderline, this physiologic uptake could have been suspected and tumors excluded in many cases. However, SPECT/CT may still be considered a determinant of the diagnosis, because most thyroid tissue residue in the neck was associated with similarly low thyroglobulin levels and was often negative on ultrasound.

Although most cases true-positive for metastases on SPECT/CT corresponded to high thyroglobulin serum levels, in some patients the procedure showed metastases when thyroglobulin levels were only slightly elevated or borderline. Not only in SPECT/CT true-positive patients were some lesions negative on ultrasound, CT, and bone scanning.

SPECT/CT rendered superfluous further imaging procedures in 2 patients, correctly identifying a case of thymic hyperplasia and a case of acute bronchitis in recovery. In the latter, the marked bilateral uptake in the bronchial tree seen on planar imaging and suspected (despite undetectable thyroglobulin levels) to represent pulmonary metastases was probably due to iodine excretion in the inflammatory exudate, as also reported by other authors who had a patient with bronchiectasis (32).

Both SPECT and low-dose CT were performed in the present study with the patient positioned identically using special cushions, markedly reducing positioning artifacts. Except for the few cases that were completely negative on planar imaging, the foci in the different regions were defined on planar imaging and not on SPECT/CT to avoid attenuation correction artifacts that may occur with the latter. 
The SPECT/CT system used in the present study exposes patients only slightly to radiation; however, the procedure, lacking the resolution of diagnostic CT, is of low diagnostic value even if the system sufficiently defines the anatomic sites of foci. In some cases, however, conventional imaging such as ultrasound, CT, and MRI may be necessary, particularly for patients in whom vascular structures must be outlined before surgery, bearing in mind that diagnostic $\mathrm{CT}$ with contrast material is not indicated when patients are to undergo radioiodine therapy shortly afterward.

In our experience, SPECT/CT represents a useful diagnostic noninvasive imaging procedure that is simple to perform and provides excellent images that, with training, are easy to read.

Thus, we suggest that SPECT/CT be used more widely in the follow-up of thyroidectomized DTC patients, particularly when planar ${ }^{131}$ I whole-body imaging is not conclusive. However, further studies of larger series of patients are needed to better establish which patients with metastases might benefit most from this new procedure, the use of which is progressively growing in nuclear medicine departments in numerous fields of clinical interest.

\section{CONCLUSION}

SPECT/CT improves the interpretation of planar ${ }^{131} \mathrm{I}$ whole-body images, precisely localizing and characterizing thyroid tissue residue and iodine-avid metastases, differentiating them from areas of physiologic uptake, reducing false-positive results on planar imaging, and thus identifying tumors more accurately. SPECT/CT can correctly modify the disease classification defined by planar imaging and allow for changes in therapeutic management, besides proving useful for establishing the therapeutic dose of radioiodine and for guiding surgical planning.

\section{REFERENCES}

1. Sherman SI. Thyroid carcinoma. Lancet. 2003;361:501-511.

2. Simpson WJ, Panzarella T, Carruthers JS, Gospodarowicz MK, Sutcliffe SB. Papillary and follicular thyroid cancer: impact of treatment in 1578 patients. Int J Radiat Oncol Biol Phys. 1998;14:1063-1075.

3. Spies WG, Wojtowicz CH, Spies SM, Shah AY, Zimmer AM. Value of posttherapy whole-body 131-I imaging in the evaluation of patients with thyroid carcinoma having undergone high-dose 131-I therapy. Clin Nucl Med. 1989; 14:793-800.

4. van Sorge-van Boxtel RA, van Eck-Smit BL, Goslings BM. Comparison of serum thyroglobulin, ${ }^{131} \mathrm{I}$ and ${ }^{201} \mathrm{Tl}$ scintigraphy in the postoperative follow-up of differentiated thyroid cancer. Nucl Med Commun. 1993;14: 365-372.

5. Lubin E, Mechlis-Frish S, Zatz S, et al. Serum thyroglobulin and iodine-131 whole-body scan in the diagnosis and assessment of treatment for metastatic differentiated thyroid carcinoma. J Nucl Med. 1994;35:257-262.

6. Sutter CW, Masilungan BG, Stadalnik RC. False-positive results of I-131 whole-body scans in patients with thyroid cancer. Semin Nucl Med. 1995;25: 279-282.

7. Franceschi M, Kusić Z, Franceschi D, Lukinac L, Roncević S. Thyroglobulin determination, neck ultrasonography and iodine-131 whole-body scintigraphy in differentiated thyroid carcinoma. J Nucl Med. 1996;37:446-451.
8. Filesi M, Signore A, Ventroni G, Melacrinis FF, Ronga G. Role of initial iodine131 whole-body scan and serum thyroglobulin in differentiated thyroid carcinoma metastases. J Nucl Med. 1998;39:1542-1546.

9. Lind P, Kohlfürst S. Respective roles of thyroglobulin, radioiodine imaging, and positron emission tomography in the assessment of thyroid cancer. Semin Nucl Med. 2006;36:194-205.

10. Mitchell G, Pratt BE, Vini L, McCready VR, Harmer CL. False positive ${ }^{131} \mathrm{I}$ whole body scans in thyroid cancer. Br J Radiol. 2000;73:627-635.

11. Leitha T, Staudenherz A. Frequency of diagnostic dilemmas in ${ }^{131} \mathrm{I}$ whole body scanning. Nuklearmedizin. 2003;42:55-62.

12. Shapiro B, Rufini V, Jarwan A, et al. Artifacts, anatomical and physiological variants, and unrelated diseases that might cause false-positive whole-body 131-I scans in patients with thyroid cancer. Semin Nucl Med. 2000;30:115-132.

13. Miyamoto S, Kasagi K, Misaki T, Alam MS, Konishi J. Evaluation of technetium-99m-MIBI scintigraphy in metastatic differentiated thyroid carcinoma. J Nucl Med. 1997;38:352-356.

14. Lind P, Gallowitsch HJ, Langsteger W, Kresnik E, Mikosch P, Gomez I. Technetium-99m-tetrofosmin whole-body scintigraphy in the follow-up of differentiated thyroid carcinoma. J Nucl Med. 1997;38:348-352.

15. Alam MS, Kasagi K, Misaki T, et al. Diagnostic value of technetium- $99 \mathrm{~m}$ methoxyisobutyl isonitrile ( $\left.{ }^{99 \mathrm{~m}} \mathrm{Tc}-\mathrm{MIBI}\right)$ scintigraphy in detecting thyroid cancer metastases: a critical evaluation. Thyroid. 1998;8:1091-1100.

16. Unal S, Menda Y, Adalet I, et al. Thallium-201, technetium-99m-tetrofosmin and iodine-131 in detecting differentiated thyroid carcinoma metastases. J Nucl Med. 1998;39:1897-1902.

17. Spanu A, Schillaci O, Madeddu G. ${ }^{99 \mathrm{~m}}$ Tc labelled cationic lipophilic complexes in malignant and benign tumors: the role of SPET and pinhole-SPET in breast cancer, differentiated thyroid carcinoma and hyperparathyroidism. $Q \mathrm{~J} \mathrm{Nucl} \mathrm{Med}$ Mol Imaging. 2005;49:145-169.

18. Feine U, Lietzenmayer R, Hanke JP, Held J, Wöhrle H, Müller-Schauenburg W. Fluorine-18-FDG and iodine-131-iodide uptake in thyroid cancer. J Nucl Med. 1996;37:1468-1472.

19. Nahas Z, Goldenberg D, Fakhry C, et al. The role of positron emission tomography/computed tomography in the management of recurrent papillary thyroid carcinoma. Laryngoscope. 2005;115:237-243.

20. Palmedo H, Bucerius J, Joe A, et al. Integrated PET/CT in differentiated thyroid cancer: diagnostic accuracy and impact on patient management. J Nucl Med. 2006;47:616-624.

21. Zoller M, Kohlfuerst S, Igerc I, et al. Combined PET/CT in the follow-up of differentiated thyroid carcinoma: what is the impact of each modality? Eur $J$ Nucl Med Mol Imaging. 2007;34:487-495.

22. Freudenberg LS, Frilling A, Kühl H, et al. Dual-modality FDG-PET/CT in follow-up of patients with recurrent iodine-negative differentiated thyroid cancer. Eur Radiol. 2007;17:3139-3147.

23. Keidar Z, Israel O, Krausz Y. SPECT/CT in tumor imaging: technical aspects and clinical applications. Semin Nucl Med. 2003;33:205-218.

24. Pérault C, Schvartz C, Wampach H, Liehn JC, Delisle MJ. Thoracic and abdominal SPECT-CT image fusion without external markers in endocrine carcinomas. The Group of Thyroid Tumoral Pathology of Champagne-Ardenne. J Nucl Med. 1997;38:1234-1242.

25. Yamamoto Y, Nishiyama Y, Monden T, Matsumura Y, Satoh K, Ohkawa M. Clinical usefulness of fusion of ${ }^{131} \mathrm{I}$ SPECT and CT images in patients with differentiated thyroid carcinoma. J Nucl Med. 2003;44:1905-1910.

26. Even-Sapir E, Keidar Z, Sachs J, et al. The new technology of combined transmission and emission tomography in evaluation of endocrine neoplasms. J Nucl Med. 2001;42:998-1004.

27. Ruf J, Lehmkuhl L, Bertram H, et al. Impact of SPECT and integrated low-dose CT after radioiodine therapy on the management of patients with thyroid carcinoma. Nucl Med Commun. 2004;25:1177-1182.

28. Tharp K, Israel O, Hausmann J, et al. Impact of ${ }^{131}$ I-SPECT/CT images obtained with an integrated system in the follow-up of patients with thyroid carcinoma. Eur J Nucl Med Mol Imaging. 2004;31:1435-1442.

29. Ingui CJ, Shah NP, Oates ME. Endocrine neoplasm scintigraphy: added value of fusing SPECT/CT images compared with traditional side-by-side analysis. Clin Nucl Med. 2006;31:665-672.

30. d'Amico A, Szczucka K, Borys D, Gorczewski K, Steinhof K. SPECT-CT fusion: a new diagnostic tool for endocrinology. Endokrynol Pol. 2006;57(suppl A):71-74.

31. Aqueveque AC, González EP, Gutiérrez BD, et al. Fusion of SPECT with computed tomography or magnetic resonance for the interpretation of abnormal tracer uptake. Rev Med Chil. 2007;135:725-734.

32. Jong I, Taubman K, Schlicht S. Bronchiectasis simulating pulmonary metastases on iodine-131 scintigraphy in well-differentiated thyroid carcinoma. Clin Nucl Med. 2005;30:688-689. 